\title{
VLBI Imaging and Astrometry of the RS CVn Binary Star IM Pegasi
}

\author{
D. E. Lebach, M. I. Ratner, and I. I. Shapiro
}

Harvard-Smithsonian Center for Astrophysics, 60 Garden St., Cambridge, MA 02138, USA

R. R. Ransom, N. Bartel, and M. F. Bietenholz

Department of Physics and Astronomy, York University, 4700 Keele St., Toronto, ON, M3J 1P3, Canada

\section{J.-F. Lestrade}

Observatoire de Paris, Place Jules Janssen, Meudon Principal Cedex, F-92195, France

\begin{abstract}
We have made $3.6 \mathrm{~cm}$ VLBI observations of the RS CVn binary star IM Pegasi (HR 8703) approximately four times per year since 1997 in support of the NASA/Stanford Relativity Gyroscope Experiment (Gravity Probe B). Phase-referenced maps reveal structural changes in the radio emission of the star on hour time scales during several of the sessions. Analyses of the VLBI phase delays with a Kalman-filter estimator reveal submilliarcsecond motions of the radio centroid of the star on hour and even subhour time scales. The observed structural changes and centroid motions often coincide with rapid changes in the star's flux density, as measured with the VLA. We report on our latest results and summarize our findings to date.
\end{abstract}

\section{Introduction}

Since 1997 we have made VLBI observations of the RS Canum Venaticorum binary star IM Pegasi (HR 8703) at the rate of about four observing sessions per year in support of the NASA/Stanford Relativity Gyroscope Experiment, Gravity Probe B (GP-B). IM Peg is the positional reference on the sky (i.e., "guide star") for GP-B, which is designed to test general relativity via measurement of the predicted motional (or "frame-dragging") and geodetic precessions of Earthorbiting gyroscopes (see, e.g., Turneaure, Everitt, \& Parkinson 1986). The goal of our VLBI observations is to determine the proper motion of IM Peg with a standard error $<0.15$ mas $\mathrm{yr}^{-1}$ relative to an extragalactic reference frame. We anticipate that such measurement accuracy will be needed for the GP-B mission to measure the precessional effects to within 0.4 mas yr$^{-1}$, or $1 \%$ of the predicted motional precession $(<0.01 \%$ of the predicted geodetic precession).

Our first session of VLBI observations, made on 1997 January 16-17, revealed an apparent motion in the radio position of IM Peg of about 0.9 mas over 
a $1.4 \mathrm{~h}$ period in which there was also a dramatic rise in flux density (Lebach et al. 1999). Such position changes are more rapid than can be explained by proper motion, parallax, and orbital motion and thus suggest physical evolution of the stellar radio emission on hour and even subhour time scales. Here we report on additional VLBI observations which show that the radio emission from this stellar system commonly undergoes structural changes on hour time scales.

\section{Observations and Data Analysis}

We had 13 sessions of $3.6 \mathrm{~cm}$ VLBI observations of IM Peg between 1997 January and 1999 December. We used the phased VLA and all ten VLBA antennas for all sessions; the NASA $70 \mathrm{~m}$ Deep Space Network antennas at Goldstone (USA), Robledo (Spain), and Tidbinbilla (Australia) and the $100 \mathrm{~m}$ antenna in Effelsberg (Germany) for most sessions; and the $46 \mathrm{~m}$ antenna in Algonquin Park (Canada) and the $43 \mathrm{~m}$ antenna in Green Bank (USA) for the first two sessions. The data from all sessions were correlated at the National Radio Astronomy Observatory (NRAO) facility in Socorro (USA).

Our observations sequenced through extragalactic radio sources $2251+158$ (3C 454.3) and $2250+194$ in addition to IM Peg to reduce greatly the sensitivity of our astrometric results to model errors. (Sources $2251+158$ and $2250+194$ are, respectively, 0.7 and 2.9 away on the sky from IM Peg, in nearly opposite directions.) The cycle time over which we repeatedly observed the three sources was $\sim 7 \mathrm{~min}$ for the first two sessions and $\sim 5.5 \mathrm{~min}$ for the remaining sessions.

After performing the usual data calibration steps with NRAO's AIPS software package, we estimated astrometric positions of IM Peg relative to $2251+158$ using two different approaches: (i) phase-referenced mapping in AIPS; and (ii) phase connection (i.e., resolution of the " $2 \pi n$ " phase ambiguities) followed by model fitting of the phase data with a least-squares Kalman-filter estimator (Herring, Davis, \& Shapiro 1990) that allows us to model atmospheric delays at zenith and station clock drifts as Gauss-Markov stochastic processes.

\section{Results}

Many RS CVn binary stars are known to have highly variable radio flux densities on hour and subhour time scales (e.g., Hjellming 1988; Jones et al. 1996). IM Peg is certainly one such binary: the $3.6 \mathrm{~cm}$ total flux density from IM Peg, as measured with the phased VLA, exceeded $10 \mathrm{mJy}$ during at least some portion of nine of our 13 sessions, and in every one of those nine the maximum flux density recorded during the session exceeded the minimum by at least $60 \%$ (and for all but two of those nine sessions by over $100 \%$ ).

Sequences of "snapshot" phase-referenced maps of IM Peg, in which each map is based on $a \sim 1 \mathrm{~h}$ subset of data, reveal structural changes on hour time scales during several of the nine radio-bright sessions. Figure 1 shows such changes over $3 \mathrm{~h}$ of our 1999 September 18-19 observations. The total flux density of IM Peg fluctuated between 24 and $26 \mathrm{mJy}$ in the two hours corresponding to the first two images and then dropped steadily to $19 \mathrm{mJy}$ in the hour corresponding to the third image. We suspect that the two radio components seen in all three maps correspond to two regions near the IM Peg primary, which has 

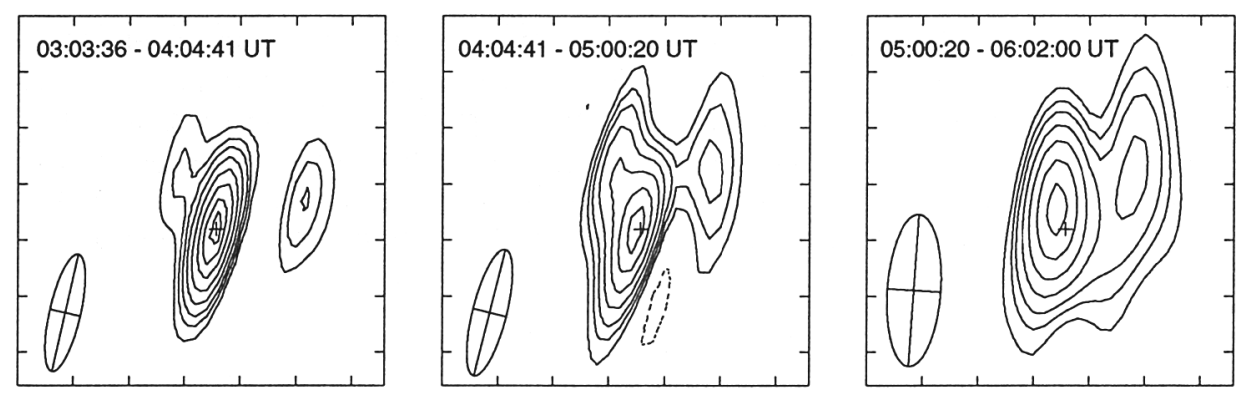

Figure 1. Phase-referenced maps of IM Peg over successive $\sim 1 \mathrm{~h}$ periods on 1999 September 19. North is up and east is to the left. Each tick mark on the boxes represents 1 mas. The contours are drawn at -1.5 (dashed) $1.5,2.5,3.5,5.0,7.0,9.5,12.0$, and $14.0 \mathrm{mJy}$ per beam area. The FWHM restoring beam for each map is shown in its lower left corner. The small cross in each map marks the position of the flux-density peak in the first map.

an estimated angular diameter of 1.3 mas (Lebach et al. 1999). Alternatively, one of the radio components could correspond to a region near the primary and the other to a region near the secondary, which to date is optically undetected.

Our Kalman-filter estimator does not produce images of IM Peg, but it can reliably discern position changes of the radio centroid on shorter time scales and of smaller magnitude than the phase-referenced maps can reliably indicate. Motion of IM Peg radio emission, as estimated with our Kalman-filter technique, often coincides with changes in radio flux density. Of the eight sessions between 1997 January and 1998 September in which $3.6 \mathrm{~cm}$ flux density of IM Peg exceeded $10 \mathrm{mJy}$, we have reliable $(>3 \sigma)$ detections of such motion in three of our sessions and marginal $(2-3 \sigma)$ detections in an additional four. The size of these motions ranges from about 0.2 to 1.2 mas. As an example, Figure 2 shows IM Peg position vs. time for the 1997 November 29-30 observing session.

To estimate the proper motion of IM Peg, we use one time-averaged position estimate from each session of VLBI observations. If we fit a model for position, proper motion, and parallax to the position estimates derived from phase-referenced maps for all sessions of observations between 1997 and 1999, the postfit RMS deviation is 0.50 mas in right ascension and 0.61 mas in declination. We believe that the deviations of our position estimates from the model are dominated by motions of the radio emission region relative to the IM Peg primary, not by measurement error. As yet the residuals to the model fit reveal no clear indication of a periodic motion of the radio emission with the 24.65 day orbital period of the binary.

In our final determination of IM Peg proper motion, we will include in our astrometric models parameters for proper acceleration and orbital motion. We also will add to our data set the position estimates from four sessions of astrometric VLBI observations of IM Peg made between 1991 and 1994 by J.F. Lestrade et al. (1995) in support of the Hipparcos mission. Our covariance analyses indicate that if we continue, as we have done in 2000 , to obtain four position estimates of IM Peg per year until the projected end of the GP-B 


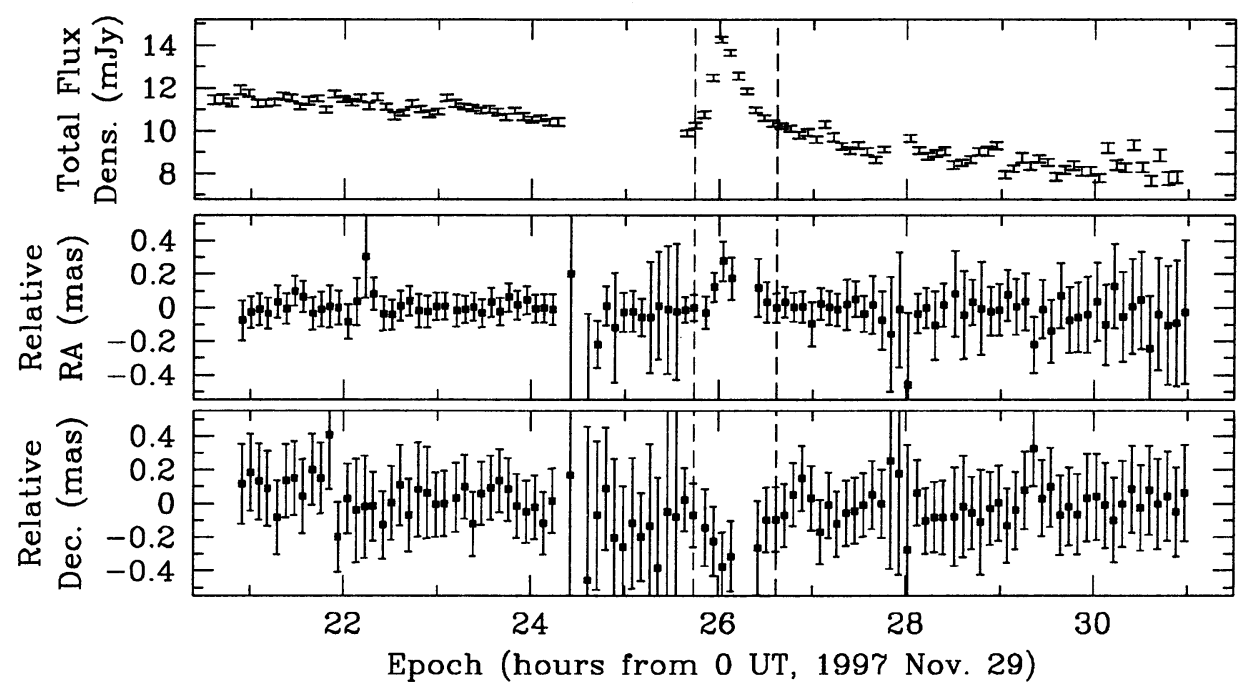

Figure 2. Total flux density at $3.6 \mathrm{~cm}$ and position estimates of IM Peg obtained with our Kalman-filter estimator for the 1997 November 29-30 VLBI observing session. The error bars are statistical standard errors. The dashed vertical lines mark the period of sharp rise and fall in total flux density. This period coincides with the only reliably detected position change seen in the figure. The statistical standard errors of the position estimates are, in general, much larger than the point-to-point scatter of the estimates because of strong correlations (due to atmospheric and instrumental effects) among position estimates closely spaced in time. These correlations also increase the statistical significance of the detected position change.

mission in about 2004 April, then we will be able to estimate the proper motion of IM Peg at the projected 2003 August midpoint of the GP-B mission with a statistical standard error of about $0.10 \mathrm{mas} / \mathrm{yr}$ in each coordinate.

\section{References}

Herring, T. A., Davis, J. L., \& Shapiro, I. I. 1990, J. Geophys. Res., 95, 12,561 Hjellming, R. M. 1988, in Galactic and Extra Galactic Radio Astronomy, eds. G. L. Verschuur \& K. I. Kellermann (2nd ed.; New York: Springer-Verlag), 381

Jones, K. L., Brown, A., Stewart, R. T., \& Slee, O. B. 1996, MNRAS, 283, 1331 Lebach, D. E., Ratner, M. I., Shapiro, I. I., Bartel, N., Bietenholz, M. F., Ransom, R. R., \& Lestrade, J.-F. 1999, ApJ, 517, L43

Lestrade, J.-F., et al. 1995, A\&A, 304, 182

Turneaure, J. P., Everitt, C. W. F., \& Parkinson, B. W. 1986, in Proc. of the Fourth Marcel Grossmann Meeting on General Relativity, ed. R. Ruffini (Amsterdam: Elsevier), 411 